\title{
Diagnósticos de enfermería al alta hospitalaria en personas con Síndrome Coronario Agudo
}

\author{
Nursing Diagnoses to Hospital Discharge in People with Acute \\ Coronary Syndrome
}

\section{Diagnósticos de enfermagem na alta hospitalar em pessoas com a síndrome coronária aguda}

\author{
Clara Inés Padilla-García, Enf., Esp., MSc* \\ Sandra Lucrecia Romero-Guevara, Enf., MSc ** \\ Fabio Alberto Camargo-Figuera, Enf., MSc., PhD *** \\ Adriana Patricia Bonilla-Marciales, Enf., Esp., MSc ****
}

\section{Resumen}

Introducción: Enfermería juega un rol fundamental en las diferentes etapas de la atención de las personas con síndrome coronario agudo, entre ellas el egreso hospitalario. Brindar cuidado acorde a las necesidades propias de estas personas, requiere de la identificación de los principales diagnósticos de enfermería. Objetivo: Determinar la prevalencia de diagnósticos de Enfermería de las personas con síndrome coronario agudo al momento del alta hospitalaria. Metodología: Estudio de corte transversal en 196 personas hospitalizadas por el evento de interés, se utilizó instrumento de valoración focalizada que evaluaba las características definitorias de los diagnósticos de enfermería: ansiedad, afrontamiento ineficaz, disposición para mejorar la religiosidad, disposición para mejorar los conocimientos, intolerancia a la actividad; los cuales fueron identificados como prioritarios según la literatura científica y por consenso de expertos. Para determinar la prevalencia de los diagnósticos se realizó análisis de definición y características definitorias. Resultados: Se evidenció como principal problema de la población de estudio los conocimientos deficientes, seguido de la intolerancia a la actividad y la ansiedad. De igual forma se logró identificar diagnósticos positivos como la disposición para mejorar los conocimientos y la religiosidad. Conclusiones: El presente trabajo nos permitió identificar necesidades reales y reconocer factores protectores al momento del alta hospitalaria en personas que han vivido un evento coronario agudo, lo cual constituye un punto de partida para el diseño de planes de cuidado y la puesta en marcha de intervenciones que conduzcan a mejorar la situación de salud de este grupo de personas. [Padilla-García Cl, Romero-Guevara SL, CamargoFiguera FA, Bonilla-Marciales AP. Diagnósticos de enfermería al alta hospitalaria en personas con Síndrome Coronario Agudo MedUNAB 2017; 20(1): 19-27].

Enfermera, Especialista en Enfermería en Cuidado Crítico, Magíster en Enfermería con Énfasis en Cuidado Cardiovascular. Docente, Programa de enfermería, Facultad de Ciencias de la Salud, UniversidadAutónoma de Bucaramanga, Floridablanca, Santander, Colombia

** Enfermera, Especialista en Atención de Enfermería en Cuidado Crítico, Magíster en Enfermería con Énfasis en Cuidado Crónico. Docente, Escuela de Enfermería, Facultad de Salud, Universidad Industrial de Santander, Bucaramanga, Santander, Colombia.

*** Enfermero, Magíster en Epidemiología, Doctor en Epidemiologia. Docente, Escuela de Enfermería, Facultad de Salud, Universidad Industrial de Santander, Bucaramanga, Santander, Colombia

**** Enfermera, Especialista en Docencia Universitaria, Magister en Educación con Énfasis en Pedagogía. Docente, Programa de Enfermería, Facultad de Ciencias de la Salud, UniversidadAutónoma de Bucaramanga, Floridablanca, Santander, Colombia

Correspondencia: Clara Inés Padilla García, Universidad Autónoma de Bucaramanga, Facultad de Ciencia de la Salud, Programa de Enfermería, Calle 157 No 14 -55, Floridablanca, Santander, Colombia. Teléfono:6436111 extensión 545. Email: cpadilla@unab.edu.co 
Palabras clave: Alta del Paciente; Diagnóstico de Enfermería; Prevalencia, Síndrome Coronario Agudo; Proceso de Enfermería.

\section{Abstract}

Introduction: Nursing plays a crucial role during the different stages of care of people with acute coronary syndrome, including hospital discharge. Providing care to these patients, according to their needs, requires the identification of the main nursing diagnoses. Objective: To determine the prevalence of nursing diagnoses of people with Acute Coronary Syndrome at the time of hospital discharge. Methodology: This is a cross-sectional study of 196 hospitalized people for this event; a focused assessment instrument was used to evaluate the defining characteristics of the nursing diagnoses: anxiety, ineffective coping of the problem, willingness to improve religiosity, readiness to improve knowledge, and intolerance to activity. These characteristics were identified as priorities according to the scientific literature and by the consensus of experts. A definition analysis and defining characteristics of the problem were performed to determine the prevalence of the diagnoses. Results: The main problem of the population studied was evidenced a deficient knowledge about the problem, followed by intolerance to activity and anxiety. Likewise, it was possible to identify some positive diagnoses such as the willingness to improve their knowledge about the problem and religiosity. Conclusions: The current study allowed identifying real needs and recognizing protective factors at the time of hospital discharge in people who have experienced an acute coronary event, which is a starting point for the design of care plans and the implementation of interventions that lead to improve the health situation of this group of people. [Padilla-García CI, Bonilla-Marciales AP, Romero-Guevara SL, Camargo-Figuera FA. Nursing Diagnoses to Hospital Discharge in People with Acute Coronary Syndrome. MedUNAB 2017; 20(1): 19-27].

Keywords: Patient Discharge; Nursing Diagnosis Prevalence; Acute Coronary Syndrome; Nursing Process.

\section{Introducción}

El síndrome coronario agudo (SCA) es un término usado para referirse a cualquier constelación de síntomas clínicos compatibles con la isquemia miocárdica aguda. Comprende infarto agudo de miocardio con elevación de ST (IAMCEST), infarto agudo de miocardio sin elevación del ST (IAMSEST) y Angina inestable (AI) -(1).

Según la Organización Mundial de la Salud (OMS) (2), la enfermedad cardiovascular es la principal causa de muerte en todo el mundo. Se calcula que en el 2012 murieron por esta causa 17.5 millones de personas, de las cuales 7.4 millones se debieron a cardiopatía coronaria.

Según el Departamento Administrativo Nacional de Estadísticas (DANE) (3), en Colombia las enfermedades

\section{Resumo}

Introdução: A enfermagem desempenha um papel fundamental nas diferentes etapas do atendimento de pessoas com síndrome coronariana aguda, incluindo alta hospitalar. Prestar cuidados de acordo com as necessidades dessas pessoas requer a identificação dos principais diagnósticos de enfermagem. Objetivo: Determinar a prevalência de diagnósticos de enfermagem de pessoas com síndrome coronariana aguda no momento da alta hospitalar. Metodologia: Foi feito um estudo de corte transversal, com 196 pessoas hospitalizadas com a síndrome coronariana e se utilizou um instrumento de avaliação enfocado nas características que definem os diagnósticos da enfermagem. ansiedade, enfrentamento ineficaz, disposição para melhorar a religiosidade, disposição para melhorar o conhecimento, intolerância à atividade; que foram identificados como prioritários de acordo com a literatura científica e por consenso de especialistas. Para determinar a prevalência dos diagnósticos, realizamos a análise da definição e das características definidoras. Resultados: O principal problema da população estudada foi o conhecimento precário, seguido da intolerância à atividade e à ansiedade. Da mesma forma, foi possível identificar diagnósticos positivos, como a vontade de melhorar o conhecimento e a religiosidade. Conclusões: o presente estudo nos permitiu identificar necessidades reais e reconhecer fatores de proteção no momento da alta hospitalar, em pessoas que sofreram um evento coronariano agudo, que é um ponto de partida para o planeação de planos de cuidados e a implementação de intervenções que levaram a melhorar a situação de saúde desse grupo de pessoas. [Padilla-García Cl, Bonilla-Marciales AP, Romero-Guevara SL, CamargoFiguera FA. Diagnósticos de enfermagem na alta hospitalar em pessoas com a síndrome coronária aguda. MedUNAB 2017; 20(1): 19-27].

Palabras-chave: Alta do Paciente; Diagnóstico de Enfermagem; Prevalência; Síndrome Coronariana Aguda; Processo de Enfermagem.

isquémicas del corazón ocupan el primer lugar de causa de muerte que corresponden a un $14.02 \%$ del total de las defunciones en el país.

En Santander la primera causa de mortalidad la constituyen los eventos cardiovasculares, seguida de las neoplasias (4). En el 2010 las enfermedades isquémicas del corazón correspondieron a $16.4 \%(5)$.

Posterior al desarrollo de un evento coronario se hace necesaria la implementación de medidas terapéuticas que minimicen el riesgo de volver a presentar un segundo evento o a presentar reingresos hospitalarios. Dentro de las estrategias de manejo, además de la aplicación de medidas terapéuticas acordes a los nuevos avances terapéuticos, se requieren cambios en el estilo de vida y el fortalecimiento en la adherencia al régimen terapéutico. Si bien es cierto que lograr estos cambios depende de muchos factores, un buen 
punto de partida es identificar cuáles son las necesidades y los aspectos claves a intervenir en las personas que han presentado un SCA, quienes se ven enfrentadas a retornar a sus hogares y retomar las actividades cotidianas y laborales, en muchos de los casos sin conocimientos, con miedos y sin la preparación para los cambios y cuidados que deben ser tenidos en cuenta para una adecuada recuperación.

Dentro del equipo interdisciplinario, el profesional de enfermería juega un rol fundamental en el cuidado de las personas que tienen o están en riesgo de sufrir enfermedades cardiovasculares. Enfermería cuenta con una herramienta denominada Proceso de Atención de Enfermería (6), que le permite de manera estructurada planear el cuidado que brinda, basado en la enunciación de diagnósticos, establecimiento de metas por medio de etiquetas de resultado y formulación de intervenciones; lo cual se realiza a través de clasificaciones estandarizadas para diagnósticos de enfermería de la North American Nursing Diagnosis Association (NANDA) (7), resultados esperados según la Clasificación de Resultados de Enfermería (CRE) (8) y Clasificación de Intervenciones de Enfermería (CIE)(9).

Los diagnósticos enfermeros son interpretaciones científicas procedentes de los datos de valoración que se usan para guiar en la planificación, implementación y evaluación del cuidado (6). Para identificar un diagnóstico de enfermería es preciso que el profesional inicie con una valoración completa y el análisis de datos de la historia clínica. En la recolección de datos es importante contar con instrumentos de valoración propios de la profesión, que permitan identificar las características definitorias o evidencias clínicas (signos y síntomas); es decir, todas aquellas manifestaciones que son observables y no observables y que van a permitir identificar los diagnósticos, que en últimas van a orientar la selección de intervenciones a realizar para alcanzar la satisfacción de cuidar de manera integral a las personas en riesgo de enfermar o que están enfermas.

La NANDA 2015-2017(7) cuenta con 235 diagnósticos de enfermería que incluyen diagnósticos reales y potenciales, los cuales cuentan con características definitorias o factores relacionados que permiten confirmar la presencia del diagnóstico en las personas que se encuentran bajo el cuidado de Enfermería.

De acuerdo a la literatura revisada, los trabajos encontrados se han enfocado principalmente a en la atención del episodio agudo, donde las necesidades predominantes son fisiológicas y se ha dado especial importancia a la ansiedad, temor y depresión. Para el momento del egreso hospitalario no se encuentran definidas las necesidades de dicha población y a su vez, no se cuenta con instrumentos propios que permitan evaluar la presencia de las características que definen los diagnósticos de enfermería. Poder identificar las necesidades prioritarias permitirá a las enfermeras establecer planes de cuidado específicos, intervenciones prioritarias y finalmente evaluar los resultados de la implementación de dicho plan para esta población, demostrando la eficacia, efectividad o la eficiencia de sus intervenciones. El objetivo de este estudio fue determinar la prevalencia de los diagnósticos de enfermería en las personas con síndrome coronario agudo al momento del alta hospitalaria.

\section{Metodología}

Estudio de corte transversal en 196 personas que estuvieron hospitalizados por SCA en tres instituciones de tercer y cuarto nivel de atención en salud de la ciudad de Bucaramanga y su área metropolitana, que presentaron alta hospitalaria a partir de diciembre de 2014 hasta completar el tamaño de la muestra en marzo de 2016. Para el cálculo de la muestra se tuvo en cuenta una aproximación de población disponible de 400 personas con SCA que egresaron de los servicios de hospitalización teniendo en cuenta estadísticas del segundo semestre de 2012, prevalencia esperada del $50 \%$ para los diagnósticos de enfermería, nivel de confianza del $95 \%$ y un error alfa de 0.05 .

Los criterios de inclusión fueron: persona mayor de 18 años, diagnóstico confirmado de SCA y en alta hospitalaria, que dominara el idioma español y que aceptara la participación y firma del consentimiento informado; el criterio de exclusión fue paciente con incapacidad para responder el cuestionario.

El presente estudio se desarrolló en 2 fases: una primera fase de diseño del formato de valoración y validación de contenido por expertos, realizando los ajustes del mismo; y una segunda fase relacionada con la aplicación del instrumento y recolección de la información en personas que cumplieran con los criterios de inclusión.

Para el diseño del instrumento y la selección de los diagnósticos, se consideraron los hallazgos en la literatura científica respecto a los problemas de salud más frecuentes en la población de interés y la experiencia de los investigadores en el área. Se evidenció que las necesidades más frecuentes de la población de estudio, estaban relacionadas con características definitorias de los diagnósticos de enfermería: intolerancia a la actividad, ansiedad, afrontamiento ineficaz, disposición para mejorar los conocimientos, conocimientos deficientes, temor, trastorno del patrón del sueño, gasto cardiaco disminuido, disposición para mejorar la religiosidad según la clasificación de diagnósticos enfermeros NANDA 2012 2014(10).

Teniendo en cuenta esta información se construyó el instrumento, el cual contenía la definición conceptual de cada uno de los diagnósticos, las características definitorias, las preguntas para la evaluación de cada una de las características y la definición operacional de cada uno de los ítems. 
Para la validación de contenido, los expertos debían evaluar el grado de pertinencia, grado de relevancia de los ítems, suficiencia de cada característica definitoria y claridad en la redacción del lenguaje empleado en cada pregunta; además, de un espacio abierto para observaciones adicionales en cada una de las características.

Después de la validación de expertos se ajustaron los ítems de acuerdo a las recomendaciones y finalmente el instrumento contempló 113 preguntas propias que facilitaban el hallazgo de características definitorias para los diagnósticos de enfermería Intolerancia a la Actividad, Ansiedad, Afrontamiento Ineficaz, Disposición para Mejorar los Conocimientos, Conocimientos Deficientes y Disposición para Mejorar la Religiosidad. Adicionalmente, se incluyó en el instrumento variables sociodemográficas y variables clínicas relacionadas con el evento coronario. Se realizó una prueba piloto con 30 participantes, la cual permitió realizar ajustes al instrumento, principalmente relacionados con el lenguaje de algunas preguntas.

La base de datos fue elaborada en Epidata 3.1, se realizó doble digitación para control de calidad de datos. Los datos se analizaron mediante el programa STATA v12. El plan de análisis consintió primero en describir las características sociodemográficas y clínicas de los participantes del estudio; se utilizaron medidas de tendencia central y dispersión para las variables continuas, para las variables categóricas se utilizaron frecuencias absolutas y relativas. La prevalencia de los diagnósticos de enfermería de interés fue calculada con sus respectivos intervalos de confianza del $95 \%$.

La presente investigación se realizó teniendo en cuenta las normas científicas, técnicas y administrativas para la investigación en salud establecidas por la Resolución número 8430 de 1993, el estudio fue aprobado por el comité de ética y fue clasificado como una investigación sin riesgo; todas las personas que aceptaron participar en el estudio dieron y firmaron consentimiento informado.

\section{Resultados}

Un total de 196 pacientes fueron estudiados, quienes presentaron una mediana de edad de 65 años con un rango entre 32 y 91 años. Una de las instituciones de carácter privado, denominada como la número 2 , fue la de mayor número de usuarios con SCA con 94 pacientes (47.96\%). En cuanto a las características sociodemográficas el $63.27 \%$ fueron hombres, el $53.06 \%$ pertenecían al régimen contributivo, es importante aclarar que el 100\% de los usuarios atendidos en la institución tres (carácter público), pertenecían al régimen subsidiado. Del total de usuarios, el $65.31 \%$ eran de nivel socioeconómico bajo (estrato uno y dos), el $64.29 \%$ tenían nivel educativo primaria o ninguna, el $59.18 \%$ eran casados y $34.69 \%$ eran independientes en su ocupación, (Tabla 1).
Tabla 1. Características sociodemográficas

\begin{tabular}{|c|c|c|}
\hline Variable & $\mathbf{N}$ & $\%$ \\
\hline \multicolumn{3}{|l|}{ Institución } \\
\hline 1 Privada & 24 & 12.24 \\
\hline 2 Privada & 94 & 47.96 \\
\hline 3 Pública & 78 & 39.80 \\
\hline \multicolumn{3}{|l|}{ Género } \\
\hline Femenino & 72 & 36.73 \\
\hline Masculino & 124 & 63.27 \\
\hline \multicolumn{3}{|l|}{ Seguridad Social } \\
\hline Contributiva & 104 & 53.06 \\
\hline Subsidiada & 92 & 46.94 \\
\hline \multicolumn{3}{|l|}{ Nivel Socioeconómico } \\
\hline Uno & 82 & 41.84 \\
\hline Dos & 46 & 23.47 \\
\hline Tres & 50 & 25.51 \\
\hline Cuatro & 14 & 7.14 \\
\hline Cinco & 1 & 0.51 \\
\hline No sabe/no responde & 3 & 1.53 \\
\hline \multicolumn{3}{|l|}{ Escolaridad } \\
\hline Ninguna & 32 & 16.33 \\
\hline Primaria & 94 & 47.96 \\
\hline Secundaria & 21 & 10.71 \\
\hline Universidad & 24 & 12.24 \\
\hline Postgrado & 21 & 10.71 \\
\hline No sabe/no responde & 4 & 2.04 \\
\hline \multicolumn{3}{|l|}{ Estado Civil } \\
\hline Casado & 116 & 59.18 \\
\hline Soltero & 25 & 12.76 \\
\hline Divorciado & 6 & 3.06 \\
\hline Viudo & 27 & 13.78 \\
\hline Separado & 5 & 2.55 \\
\hline Unión libre & 13 & 6.63 \\
\hline No sabe/no responde & 4 & 2.04 \\
\hline \multicolumn{3}{|l|}{ Ocupación } \\
\hline Empleado/a & 27 & 13.78 \\
\hline Independiente & 68 & 34.69 \\
\hline Ama de casa & 58 & 39.59 \\
\hline Pensionado & 32 & 16.33 \\
\hline Otro & 7 & 3.57 \\
\hline No sabe/no responde & 4 & 2.04 \\
\hline
\end{tabular}


En relación con los problemas médicos existentes asociados al evento, el $83.16 \%$ eran hipertensos y el $28.57 \%$ diabéticos. En cuanto a antecedentes u otros factores de riesgo cardiovascular el más prevalente fue la dislipidemia con un $43.88 \%$, seguido de tabaquismo con un $33.16 \%$, el sedentarismo y la obesidad fueron los menos prevalentes con un $20.41 \%$ y $19.9 \%$ respectivamente. El $27.55 \%$ tenía antecedente de angina inestable o IAM, (Tabla 2).

En cuanto a variables relacionadas con el evento, la mediana de la estancia hospitalaria fue de 7 días, con un rango de 1 a 72 días; respecto a la fracción de eyección se encontró que la mediana fue de $52.5 \%$ con un rango entre $14 \%$ y $76 \%$. Al clasificar el evento coronario agudo, el
IAMSEST fue el más frecuente, seguido de la angina inestable, con un $42.35 \%$ y $39.29 \%$ respectivamente. Es importante aclarar que algunos pacientes que fueron ingresados con diagnóstico de angina estable o inestable progresaron a infarto agudo de miocardio. De 95 pacientes en quienes se reportó el Killip, el 74.74\% fue clasificado como I, (Tabla 2).

Respecto al tipo de intervención realizada, el manejo médico fue el más frecuente con un $62.75 \%$, seguido de la angioplastia con un $32.14 \%$; de los pacientes que recibieron manejo médico, al $34.69 \%$ le realizaron cateterismo cardiaco diagnóstico, (Tabla 2).

Tabla 2. Características clínicas

\begin{tabular}{|c|c|c|}
\hline Variable & $\mathbf{N}$ & $\%$ \\
\hline \multicolumn{3}{|l|}{ Diagnóstico médico } \\
\hline IAMCEST & 33 & 16.84 \\
\hline IAMSEST & 83 & 42.35 \\
\hline Angina Inestable & 77 & 39.29 \\
\hline Angina Estable & 3 & 1.53 \\
\hline \multicolumn{3}{|l|}{ Clasificación Killip } \\
\hline Killip I & 71 & 74.74 \\
\hline Killip II & 12 & 12.63 \\
\hline Killip III & 8 & 8.42 \\
\hline Killip IV & 4 & 4.21 \\
\hline \multicolumn{3}{|l|}{ Antecedente deenfermedad coronaria } \\
\hline Angina Inestable Preexistente & 23 & 11.73 \\
\hline IAM Preexistente & 31 & 15.82 \\
\hline \multicolumn{3}{|l|}{ Comorbilidades y factores de riesgo } \\
\hline Hipertensión Arterial & 163 & 83.16 \\
\hline Diabetes Mellitus & 56 & 28.57 \\
\hline Tabaquismo & 65 & 33.16 \\
\hline Sedentarismo & 40 & 20.41 \\
\hline Obesidad & 39 & 19.9 \\
\hline Dislipidemia & 86 & 43.88 \\
\hline \multicolumn{3}{|l|}{ Intervenciones realizadas } \\
\hline Manejo médico con cateterismo cardiaco & 68 & 34.69 \\
\hline Solo manejo médico & 55 & 28.06 \\
\hline Angioplastia coronaria & 63 & 32.14 \\
\hline Revascularización miocárdica & 10 & 5.10 \\
\hline
\end{tabular}


Tabla 3. Prevalencia de los diagnósticos de Enfermería de interés en la muestra.

\begin{tabular}{lccc}
\hline Diagnóstico de enfermería & $n=196$ & $\%$ & IC95\% \\
\hline Intolerancia a la actividad & 121 & 61.70 & $54.50-68.50$ \\
\hline Disposición para mejorar la religiosidad & 128 & 65.30 & $58.10-71.90$ \\
\hline Afrontamiento ineficaz & 39 & 19.90 & $14.50-26.10$ \\
\hline $\begin{array}{l}\text { Disposición para mejorar los } \\
\text { conocimientos }\end{array}$ & 191 & 97.40 & $94.10-9.910$ \\
\hline Conocimientos deficientes & 159 & 81.10 & $74.90-86.30$ \\
\hline Ansiedad & 69 & 35.20 & $28.50-42.30$ \\
\hline
\end{tabular}

En relación con el objetivo del estudio, los diagnósticos de enfermería más prevalentes fueron Disposición para Mejorar los Conocimientos con un $97.40 \%$ y Conocimientos Deficientes con un $81.10 \%$; de otra parte, los menos frecuentes fueron Ansiedad y Afrontamiento Ineficaz con un $35.20 \%$ y $19.90 \%$ respectivamente, (Tabla 3 ).

\section{Discusión}

Los diagnósticos de enfermería más prevalentes correspondieron al dominio de Cognición y Percepción, específicamente Disposición para Mejorar los Conocimientos y Conocimientos Deficientes, seguido del dominio Tolerancia y Afrontamiento al Estrés, con los diagnósticos Afrontamiento Ineficaz y Ansiedad; en el dominio de Principios Vitales se identificó el diagnóstico Disposición para Mejorar la Religiosidad y finalmente en el dominio de Actividad y Reposo el diagnóstico de Intolerancia a la Actividad. A partir de estos resultados la enfermera podrá establecer planes de cuidado adecuados y específicos a las necesidades de esta población, aplicando intervenciones y resultados propios de la profesión.

De acuerdo a los hallazgos de la literatura revisada el SCA es más prevalente en hombres, en un estudio de diferencias según sexo en el tratamiento y la evolución de pacientes con SCA $74.60 \%$ de la muestra fueron varones(11).

En una revisión integrativa de estudios sobre la recuperación después de un evento cardiaco agudo, los trabajos que incluyeron tanto hombres como mujeres, el porcentaje de hombres fue mayor y estuvo entre $64.60 \%$ hasta $75.30 \%$, en comparación con $63.27 \%$ del presente trabajo. En cuanto a la edad, el promedio en años, en los trabajos que no discriminaron por género estuvo entre 59.1 a 62 años, valores ligeramente por debajo de la mediana de edad de los participantes del presente estudio que fue de 65 años(12).
En cuanto a otras características sociodemográficas y clínicas se evidencia similitud con lo reportado en general en la literatura, particularmente en un ensayo clínico que tenía como objetivo mejorar los conocimientos, las actitudes y las creencias sobre la enfermedad en pacientes con SCA, en el cual encontramos características predominantes el estado civil casado o unión con otra persona, desempleados, sistema de salud no privado, el nivel educativo predominante fue secundaria, sin embargo, el 34.68\% tenían una educación poco formal o primaria. En el estudio mencionado, los principales factores de riesgo relacionados con el evento fueron hipercolesterolemia, hipertensión y tabaquismo, al igual que en el presente trabajo, sin embargo, en nuestro caso el principal factor de riesgo fue la hipertensión en el $83.16 \%$ de los estudiados, muy por encima del $60 \%$ en el estudio en comparación(13).

Al comparar la prevalencia de los diagnósticos de enfermería al alta hospitalaria con los hallazgos de la literatura, la mayoría de los estudios se enfocan en la caracterización de aspectos o situaciones muy específicas como la ansiedad, el temor, la fatiga, y a la identificación de complicaciones derivadas del evento como la falla cardiaca, arritmias entre otras. Igualmente la mayoría de los trabajos se realizan después del egreso hospitalario y no al momento del egreso. De otra parte, se utilizan diferentes tipos de escalas, lo cual limita la comparación.

Lo anterior hace que la presente investigación sea única en nuestro medio, favorece el uso de un lenguaje propio de la profesión de enfermería, aporta instrumentos de evaluación que contemplan las características definitorias de diversos diagnósticos de enfermería presentes al egreso hospitalario de las personas que han sufrido un SCA, lo cual contribuye al fortalecimiento disciplinar.

En relación con el diagnóstico de Enfermería Conocimientos Deficientes, presenta una prevalencia de $81.10 \%$, valor que se encuentra por encima del hallado en un programa de 
preparación y apoyo para el cuidado en el hogar en 2011 en personas con enfermedad crónica (entre ellas alteraciones cardiovasculares), donde se encontró una prevalencia de $52.80 \%$ en dicho diagnóstico (14). Por otro lado un estudio realizado en Brasil evaluaron el conocimiento de la enfermedad, en donde se encontraba que el $58.30 \%$ de los participantes presentaban enfermedad cardiaca isquémica; en éste estudio el $81 \%$ de los pacientes con diagnóstico menor a 10 años tenían conocimientos entre regulares y malos, porcentaje que se asemeja a lo encontrado en este estudio(15).

Con respecto a la Disposición para Mejorar el Conocimiento, no se encontró evidencia de que haya sido evaluado en otros estudios con similar contexto; lo anterior se explica debido a que el enfoque siempre va dirigido hacia el déficit de conocimientos. En el presente estudio los resultados dejan ver que el $97.40 \%$ de los participantes manifestaron el deseo de querer saber sobre el proceso enfermedad y su manejo. La presencia de este diagnóstico puede estar relacionada con un adecuado afrontamiento de la situación vivida, aspecto que favorece la recuperación y rehabilitación de este tipo de población y, por eso, debe ser tenido en cuenta por el profesional de enfermería no solo durante el alta hospitalaria sino durante todo el proceso de cuidado.

El diagnóstico Intolerancia a la actividad, se encuentra presente en este estudio en un $61.70 \%$ de los participantes, es preciso aclarar que la fatiga fue la principal característica definitoria, esta a su vez, es definida como un síntoma frecuente e invalidante en la fase de recuperación temprana posterior al infarto de miocardio. Un estudio de investigación de síntomas posterior a infarto de miocardio encontró que el $52 \%$ de las personas reportaban fatiga postinfarto(16). La medición de la fatiga fue hecha a través de 4 dimensiones (fatiga física, fatiga mental, reducción de la motivación y reducción de la actividad) criterios que se asemejan a la definición del Diagnóstico de enfermería Intolerancia a la Actividad de la NANDA "Insuficiente energía fisiológica y psicológica para tolerar o completar las actividades de la vida diaria requeridas o deseadas" y a las características evaluadas en el presente estudio(10).

Las personas post infarto presentan fatiga como característica (síntoma) más común con prevalencias entre en un $63 \%-76 \%(17,18)$, porcentajes muy cercanos al encontrado en este trabajo. La presencia de fatiga se constituye a su vez en un factor negativo, debido a que puede ser asociada a resultados cardiacos pobres incluyendo el aumento de readmisiones y muerte súbita cardiaca, a su vez puede persistir de $6-8$ meses posterior al evento cardiaco(17).

Con respecto a la religiosidad son pocos los estudios que han relacionado los efectos de la religiosidad y espiritualidad sobre la morbilidad y mortalidad cardiovascular (19); la presencia del diagnóstico Disposición para Mejorar la Religiosidad es un aspecto positivo para la población en estudio, ya que la relación de religiosidad y espiritualidad están asociados con resultados cardiovasculares positivos y menos factores de riesgo de enfermedad cerebrovascular. La presencia de altos niveles de religiosidad, entendido como el aumento de actividades religiosas como estrategia de afrontamiento, se ha asociado con diversos resultados psicosociales en pacientes con enfermedades del corazón, incluyendo mayor recuperación, menos depresión y ansiedad y mayor bienestar existencial(20).

En cuanto al diagnóstico Afrontamiento Ineficaz que se presentó en un bajo porcentaje de la población estudiada (19.90\%), es importante tener en cuenta que es un aspecto de gran importancia para dicha población, dado que los síntomas experimentados después cursar con un SCA pueden estar asociados con las estrategias de afrontamiento, las cuales pueden ser positivas o negativas (21). Arnorld y colaboradores (22) reportan que las personas con infarto agudo de miocardio que presentan estrés moderado a alto muestran mayor mortalidad a largo plazo.

En el presente estudio al igual que lo evidenciado en la literatura, el diagnóstico Ansiedad estuvo presente en un importante porcentaje de la población estudiada (35.20\%), se ha evidenciado que posterior a un evento cardiovascular las personas tienen impactos psicológicos y físicos, dentro de los psicológicos se pueden encontrar comúnmente la ansiedad y el temor $(23,24)$. Un estudio multicéntrico reporta que las personas post infartadas presentan altos niveles de ansiedad, encontrándose una prevalencia en promedio del $40 \%$ en comparación con poblaciones sanas que tienen prevalencias del $17 \%$ (25). Otro estudio reporta que la ansiedad en personas post infartadas fue del 19.50\% (26).

Un aspecto que no fue estudiado en el presente trabajo pero que se debe tener en cuenta, es la relación entre la ansiedad y depresión. Un estudio reporta que las personas con SCA presentan dos clases de relevancia clínica de los síntomas de depresión y ansiedad; la primera clase es denominada depresión y algunos síntomas de ansiedad, la cual fue hallada en el $76 \%$ de los pacientes y la segunda clase es denominada ansiedad y algunos síntomas de depresión encontrándose en un $24 \%$ de los pacientes(27).

En consideración con lo expuesto, la ansiedad debe ser diagnosticada y manejada en poblaciones con SCA antes del egreso hospitalario, teniendo en cuenta que se asocia con mayor riesgo de resultados adversos cardiacos en un 36\%, y se asocia a mayor mortalidad y nuevos eventos cardiacos (28).

Las personas con enfermedades cardiovasculares y específicamente las personas con SCA tienen aspectos y características propias que pueden favorecer o entorpecer el proceso de recuperación. Es por ello que el profesional de enfermería tiene como meta potenciar los aspectos 
favorables presentes, tales como la disposición para mejorar los conocimientos y la disposición para mejorar la religiosidad. De esta manera se enfoca el cuidado en la potenciación de los aspectos positivos y las necesidades reales de los pacientes superando los manejos y enseñanza netamente farmacológicos, específicamente al alta hospitalaria.

Claramente se ha establecido que el conocimiento por sí solo no garantiza la adherencia a los regímenes terapéuticos; sin embargo, se ha determinado que la adherencia terapéutica aumenta cuando se desarrolla la simplificación de los regímenes terapéuticos, se brinda educación acerca de la importancia del tratamiento y se evalúa constantemente el cumplimiento del tratamiento(29).

Estos resultados generan una gran preocupación y cuestionamiento, no solo por la divergencia entre las necesidades de los usuarios de querer conocer y lo que realmente saben, además nos permite preguntarnos acerca del rol de los diferentes profesionales de salud en la preparación de los usuarios para el cuidado en el hogar.

Para obtener mejores resultados en el cuidado de las personas que presentan enfermedades cardiovasculares, es preciso tener en cuenta diferentes recomendaciones, entre ellas las dadas por la OMS en el informe sobre la situación mundial de las enfermedades no transmisibles 2014(30). Enfermería puede aportar en la solución de la problemática y se requiere que hallazgos de este tipo de investigaciones se incorporen a la práctica, a la academia y al sistema de salud, así se podrá fortalecerse el rol y el cuidado que enfermería brinda a las personas con enfermedades cardiovasculares. Es preciso que se dé un giro al cuidado actual de este tipo población ya que se ha centrado en el control de la sintomatología, principalmente con medidas farmacológicas.

Conocer las necesidades reales de las personas post infartadas al egreso hospitalario favorece el análisis de los problemas presentes que exigen acciones específicas de enfermería; además favorece la realización de investigaciones que generen mayor conocimiento acerca de las necesidades de los pacientes y de las capacidades y conocimientos que debe tener el profesional de enfermería. Este estudio puede contribuir al desarrollo científico de la profesión así como la aplicación y generación de programas propios para el alta hospitalaria de la persona con SCA.

Es importante tener en cuenta que las acciones de cuidado dirigidas a este tipo de población no solamente deben involucrar a la persona sujeto de cuidado, sino que también se debe involucrar tanto a los cuidadores como a sus familiares con el fin de optimizar y mejorar los resultados. Conocer los diagnósticos de enfermería al egreso hospitalario permite puntualizar el tipo de intervención que requiere la población posterior a un evento cardiaco, no solamente durante el periodo agudo sino también durante los procesos de rehabilitación.
La principal fortaleza del estudio es ser un estudio multicéntrico realizado en instituciones hospitalarias de tercer y cuarto nivel de atención; de igual forma, se obtuvo una representatividad de la población hospitalaria y se caracterizó los principales diagnósticos de enfermería en personas con síndrome coronario agudo al egreso hospitalario, además de ser uno de los pocos estudios en la región para esta condición de salud.

Como limitantes se tiene que por tratarse de un estudio de tipo transversal no es posible determinar la temporalidad de la presencia de los diagnósticos observados; de otra parte, podría presentarse sesgo de información por las respuestas de los sujetos al cuestionario dirigido, dado que se presentó información faltante en algunos de los ítems evaluados. Se recomienda para futuros estudios realizar diseños de tipo prospectivo, ya sean observacionales o de intervención, que nos permita tener un mejor panorama de la prevalencia e incidencia de diagnósticos de enfermería, de igual forma realizar estudios analíticos que nos permitan observar factores asociados a los mismos, con el fin de diseñar y evaluar planes de cuidado específicos. Igualmente, proponer estudios de intervención que evalúen el efecto de las intervenciones de enfermería propuestas para los diagnósticos de enfermería aquí reportados, de manera que se pueda demostrar la eficacia y efectividad de las mismas para este tipo de pacientes con SCA en el momento del alta.

\section{Conclusiones}

Los diagnósticos más prevalentes al alta hospitalaria en pacientes con SCA fueron: Disposición para Mejorar los Conocimientos y Conocimientos Deficientes. Por otro lado, los menos frecuentes fueron: Ansiedad y Afrontamiento Ineficaz, sin dejar de ser relevantes, dadas sus implicaciones en el proceso de recuperación en un evento cardiovascular.

El diseño de planes de cuidado de los profesionales de enfermería que intervienen en el egreso y recuperación de personas que han vivido un evento coronario agudo, debe estar dirigido al manejo de los diagnósticos identificados, con la puesta en marcha de intervenciones que conduzcan a mejorar la situación de salud de este grupo de personas.

\section{Conflicto de intereses}

Los autores declaran libremente no tener conflicto de intereses.

\section{Referencias}

1. Anderson JL, Adams CD, Antman EM, Bridges CR, Califf RM, Casey DE, et al. 2011 ACCF/AHA Focused Update Incorporated Into the ACC/AHA 2007 Guidelines for the Management of Patients With Unstable 
Angina/Non-ST-Elevation Myocardial Infarction. Circulation. 10 de mayo 2011;123(18):e426-579.

2. World Health Organization. Global status report on noncommunicable diseases 2014 [Internet]. Geneva: World Health Organization; 2014 [citado el 29 de junio de 2017]. Disponible en: http://www.who.int/nmh/ publications/ncd-status-report-2014/en/

3. Departamento Administrativo Nacional de Estadística. Defunciones por grupos de edad y sexo, según departamento, municipio de residencia y grupos de causas de defunción (lista de causas agrupadas 6/67 CIE-10 de OPS) [Internet]. Bogotá, Colombia: DANE; 2011 [citado el 29 de junio de 2017]. (Defunciones No Fetales 2009). Disponible en: https://www.dane.gov.co/ index.php/estadisticas-por-tema/salud/nacimientos-ydefunciones/defunciones-no-fetales/defunciones-nofetales-2009

4. Ochoa ME, Otero JA, Hormiga CM, López L. Perfil de morbilidad y mortalidad de Santander (2010) | Observatorio de Salud Pública de Santander. Rev Obs Salud Pública Santander. 2010;5(2):3-30.

5. Departamento Administrativo Nacional de Estadística. Defunciones por grupos de edad y sexo, según departamento, municipio de residencia y grupos de causas de defunción (lista de causas agrupadas 6/67 cie-10 de ops) [Internet]. Bogotá, Colombia: DANE; 2012 [citado el 29 de junio de 2017]. (Defunciones No Fetales 2010). Disponible en: https://www.dane.gov.co/ index.php/estadisticas-por-tema/salud/nacimientos-ydefunciones/defunciones-no-fetales/defunciones-nofetales-2010

6. Cachón JM, Álvarez C, Palacios D. El significado del lenguaje estandarizado NANDA-NIC-NOC en las enfermeras de cuidados intensivos madrileñas, abordaje fenomenológico. Enferm Intensiva. 2012;23(2):68-76.

7. NANDA International, Herdman T. Diagnósticos enfermeros. Definiciones y clasificación 2015-2017. 1ra ed. Barcelona: Elsevier España, S.L.U.;2015.

8. Moorhead S, Johnson M, Maas M, Swanson E, editores. Clasificación de Resultados de Enfermería (NOC): Medición de resultados en salud. 4a Edición. Barcelona, España.: Elsevier España; 2008.

9. Bulechek GM, Butcher H, McCloskey J. Clasificación de Intervenciones de Enfermería (NIC). 5a edición. Barcelona, España.: Elsevier España, S.L.U.;2009.

10. NANDA International, Herdman T. Diagnósticos enfermeros. Definiciones y clasificación 2012-2014. 1ra ed. Barcelona: Elsevier España, S.L.U.;2012.

11. Ferraz M, Belzunegui T, Marín B, Martínez Ó, Jiménez X. Diferencias según sexo en el tratamiento y la evolución de los pacientes afectados de síndrome coronario agudo. An Sist Sanit Navar. Agosto de 2014;37(2):249-55.

12. Barnason S, Zimmerman L, Nieveen J, Schulz P, Young $L$. Patient recovery and transitions after hospitalization for acute cardiac events: an integrative review. J Cardiovasc Nurs. Abril de 2012;27(2):175-91.

13. O'Brien F, McKee G, Mooney M, O'Donnell S, Moser D. Improving knowledge, attitudes and beliefs about acute coronary syndrome through an individualized educational intervention: a randomized controlled trial. Patient Educ Couns. Agosto de 2014;96(2):179-87.

14. Guevara R, Lucrecia S, Estupiñan S, Paola J, Díaz R, Johanna L. Eficacia de las intervenciones de enfermería mediante un programa para el cuidado en el hogar. Rev Cuba Enferm. marzo de 2011;27(1):20-30.

15. C M, Delgado M, Castillo O, Prats O, Gomez D. Nivel de conocimientos sobre la enfermedad en los adultos mayores con diabetes mellitus tipo 2. Rev Cienc
Médicas Pinar Río. junio de 2011;15(2):122-32.

16. Alsén P, Brink E, Brändström Y, Karlson BW, Persson L$\mathrm{O}$. Fatigue after myocardial infarction: relationships with indices of emotional distress, and sociodemographic and clinical variables. Int J Nurs Pract. Agosto 2010;16(4): 326-34.

17. Crane PB, Efird JT, Abel WM. Fatigue in Older Adults Postmyocardial Infarction. Front Public Health [Internet]. 11 de abril de 2016;4. Disponible en: http://www. ncbi.nlm.nih.gov/pmc/articles/PMC4826886/

18. Kim HM, Kim J, Hwang SY. Health-related quality of life in symptomatic postmyocardial infarction patients with left ventricular dysfunction. Asian Nurs Res. marzo de 2015;9(1):47-52.

19. Lucchese FA, Koenig HG. Religion, spirituality and cardiovascular disease: research, clinical implications, and opportunities in Brazil. Rev Bras Cir Cardiovasc. marzo de 2013;28(1):103-28.

20. Trevino KM, McConnell TR. Religiosity and religious coping in patients with cardiovascular disease: change over time and associations with illness adjustment. J Relig Health. December 2014;53(6):1907-17.

21. Fredriksson U, Alsén P, Karlson BW, Brink E. Fatigue two months after myocardial infarction and its relationships with other concurrent symptoms, sleep quality and coping strategies. J Clin Nurs. Agosto 2015;24(15-16):2192-200.

22. Arnold SV, Smolderen KG, Buchanan DM, Li Y, Spertus JA. Perceived stress in myocardial infarction: long-term mortality and health status outcomes. J Am Coll Cardiol. 30 de octubre 2012;60(18):1756-63.

23. Bhattacharyya M, Stevenson F, Walters K. Exploration of the psychological impact and adaptation to cardiac events in South Asians in the UK: a qualitative study. BMJ Open. 8 de julio 2016;6(7):e010195.

24. Huffman JC, Celano CM, Januzzi JL. The relationship between depression, anxiety, and cardiovascular outcomes in patients with acute coronary syndromes. Neuropsychiatr Dis Treat. 2010;6:123-36.

25. Moser DK, Dracup K, Evangelista LS, Zambroski CH, Lennie TA, Chung ML, et al. Comparison of prevalence of symptoms of depression, anxiety, and hostility in elderly patients with heart failure, myocardial infarction, and a coronary artery bypass graft. Heart Lung J Crit Care. Octubre de 2010;39(5):378-85.

26. Hanssen TA, Nordrehaug JE, Eide GE, Bjelland I, Rokne B. Anxiety and depression after acute myocardial infarction: an 18-month follow-up study with repeated measures and comparison with a reference population . Eur J Cardiovasc. Diciembre de 2009;16(6):651-9.

27. Tisminetzky M, Bray BC, Miozzo R, Aupont O, McLaughlin TJ. Identifying Symptom Profiles of Depression and Anxiety in Patients with an Acute Coronary Syndrome Using Latent Class and Latent Transition Analysis. Int J Psychiatry Med. 2011;42(2):195-210.

28. Roest AM, Martens EJ, Denollet J, de Jonge P. Prognostic association of anxiety post myocardial infarction with mortality and new cardiac events: a metaanalysis. Psychosom Med. Julio 2010;72(6):563-9.

29. Salcedo A, Ochoa G, Maritza A. Degrees of risk for therapeutical adherence in persons with arterial hypertension. Av En Enferm. Enero 2014;32(1):33-43.

30. Organización Mundial de la Salud. Informe sobre la situación mundial de las enfermedades no transmisibles 2014 [Internet]. Geneva, Switzerland: World Health Organization; 2014 [citado el 4 de julio de 2017]. Disponible en: http://www.who.int/nmh/publications/ ncd-status-report-2014/es/ 